\title{
Comparaison chez l'homme de l'absorption du lactose et du lactose hydrolyse ${ }^{(1)}$
}

\author{
par \\ M. J. BEAUFRAND, B. POULLAIN, B. MUNSCH \\ et G. DEBRY
}

Parmi les dérivés du lait que les progrès de la technologie permettent d'adapter à des situations nutritionnelles particulières, le lactose hydrolysé (Roger et al., 1976) présente certaines caractéristiques intéressantes pour les sujets intolérants au lactose. Cette intolérance peut être due, soit à une déficience congénitale extrêmement rare, soit à un déficit transitoire (nourrissons, enfants) (Garga C. et al., 1976), soit à la perte progressive de l'activité lactasique de l'intestin à la suite de modifications des habitudes de consommation, en particulier chez l'adulte et le vieillard (Jones D. V. et al., 1974).

L'intolérance au lactose est définie, si l'on se réfère au test de charge en lactose $(50 \mathrm{~g})$, par une élévation de la glycémie inférieure à $250 \mathrm{mg} / 1$ au cours de l'épreuve (Sahi T., 1974). Lors d'une étude épidémiologique chez des enfants noirs américains, Paige (1975) obtint 54 p. 100 de tests au lactose positifs. Toutefois malgré cette réponse positive au test la plupart des enfants peuvent consommer $250 \mathrm{ml}$ de lait par jour sans qu'apparaissent des symptômes cliniques d'intolérance. Chez de tels sujets (Paige D. M. et al., 1975) l'administration de lait à lactose hydrolysé augmente la réponse glycémique post-prandiale.

Lorsque les sujets présentent une intolérance clinique et biologique Jones (1976) et Turner (1976) observent que l'intensité des manifestations cliniques est proportionnelle à la quantité de lactose absorbée.

Ces travaux montrent que malgré un déficit en lactase déterminé par le test d'exploration fonctionnelle au lactose l'enfant supporte bien une consommation normale de lait. Il semble donc que l'utilisation de lait à lactose hydrolysé dans l'alimentation courante chez

(1) Travail réalisé dans le cadre ỏu contrat D.G.R.S.T. n 74-7-1047, par le Département de Nutrition et des Maladies Métaboliques de l'Université de Nancy I et du groupe de recherches en Nutrition et Diététique de l'I.N.S.E.R.M. U 59 Directeur : professeur G. Debry, 40, rue Lionnois - 54000 Nancy. 
l'adolescent, l'adulte ou le vieillard aille à l'encontre du but recherché puisqu'elle accélérerait le processus de dégénérescence de l'activité lactasique de la muqueuse.

L'utilisation à des fins diététiques du lactose hydrolysé impose une étude strictement médicale, une fois défini le type de produit, son rôle et le type de malade auquel il est destiné. Son emploi à des fins alimentaires nécessite néanmoins une approche nutritionnelle des conséquences de son introduction dans la ration.

En particulier, les problèmes posés par la teneur en galactose doivent être envisagés sur un plan expérimental et en fonction de l'utilisation prévue qui conditionne les quantités ingérées. S'il est indiscutable que la galactosémie congénitale est provoquée par un déficit en galactose 1.P. uridyl-transférase entraînant une accumulation de galactose 1.P. dans le foie, les hématies, le cristallin en particulier (Bondy P. K. et al., 1974 ; Roger F. C., 1969), la responsabilité de régimes riches en galactose dans l'étiologie de la cataracte est suspectée (Seetharam Bhat K. et al., 1974). Ce point est néanmoins controversé et il semble tant chez l'animal que chez l'homme, que des déséquilibres alimentaires soient des facteurs favorisants voire déclenchants de la cataracte (Seetharam Bhat K. et al., 1974 ; Bunce G. E. et al., 1976). A l'inverse, un régime équilibré, apportant par ailleurs 20 p. 100 de calories sous forme de galactose n'a pu provoquer l'apparition de cataracte chez le rat (Heffley J. D. et al., 1974).

Chez l'enfant, certains auteurs ont émis l'hypothèse d'une corrélation entre les teneurs en galactose du régime et en galactolipides du cerveau (Varma S. N. et al., 1974). Ce point est lui aussi controversé (Waar D. H., 1975).

Dans le cadre de ce travail, nous nous sommes attachés à étudier chez des sujets sains la cinétique d'absorption du lactose hydrolysé par rapport à celle du lactose pur. Il a paru intéressant de comparer l'effet du remplacement du disaccharide par une quantité équivalente de ses sucres constitutifs (glucose et galactose) sur l'absorption des sucres et la stimulation de la sécrétion de l'insuline.

\section{MATERIEL ET METHODES}

\subsection{Préparation du lactose hydrolysé*}

L'hydrolyse du lactose contenu dans un ultrafiltrat de lait est réalisée à l'aide de la lactase provenant de Saccharomyces lactis, dans un réacteur enzymatique à membrane (Roger et al., 1976).

Dans le produit testé 85 p. 100 du lactose est hydrolysé.

* Fourni par le Laboratoire de Technologie Laitière de l'I.N.R.A. (Rennes). 
TABLEAU 1

Composition du concentré de lactose hydrolysé ( $\mathrm{g} / 100 \mathrm{~g}$ de produit $\mathrm{sec}$ )

\begin{tabular}{l|c}
\hline Protides & 2,6 \\
Cendres & 2,5 \\
Sucres totaux & 84,6 \\
Glucose & 36,6 \\
Galactcse & 32,2 \\
Lactose & 14,7 \\
\hline
\end{tabular}

\subsection{Protocole}

Trois sujets adultes en bonne santé ont absorbé le matin à jeun lors de deux épreuves semblables à $1 \mathrm{j}$ d'intervalle une dose de lactose pur et une quantité équivalente de lactose hydrolysé.

Le temps d'ingestion est identique et inférieur à $1 \mathrm{mn}$.

Ces doses correspondent approximativement à celles apportées lors d'un petit déjeuner classique par «le bol de lait » $(250 \mathrm{cc})$ :

- premier jour : $15 \mathrm{~g}$ de lactose pur en solution dans l'eau à 12,5 p. 100 ;

- deuxième jour : 23,7 g de lactose hydrolysé en solution dans l'eau à 12,5 p. 100 .

La quantité totale de sucre apportée (15 g) est identique lors des deux épreuves.

Lors de chaque test, les prélèvements de sang veineux ont été effectués aux temps suivants : à jeun, puis 20,40 et $80 \mathrm{mn}$ après l'absorption du lactose et du lactose hydrolysé.

\subsection{Méthodes}

La glycémie et la galactosémie sont déterminées à partir du sang recueilli sur fluorure de sodium et oxalate de calcium avec les méthodes suivantes : glucose-oxydase (Boehringer) adaptée à l'autoanalyseur I, et galactose-déshydrogénase (Boehringer). L'insulinémie est déterminée à partir du sang recueilli sur héparine par une méthode dérivée de Hales et Randle (1963) et de Morgan et Lazarow (1963) par utilisation des trousses de dosage du C.E.A.-C.E.N., S.O.R.I.N. en construisant les courbes étalon dans un sérum à faible teneur en insuline (Marschner, 1974). 


\section{RESULTATS}

Les résultats sont notés dans le tableau 2 et la figure 1 .
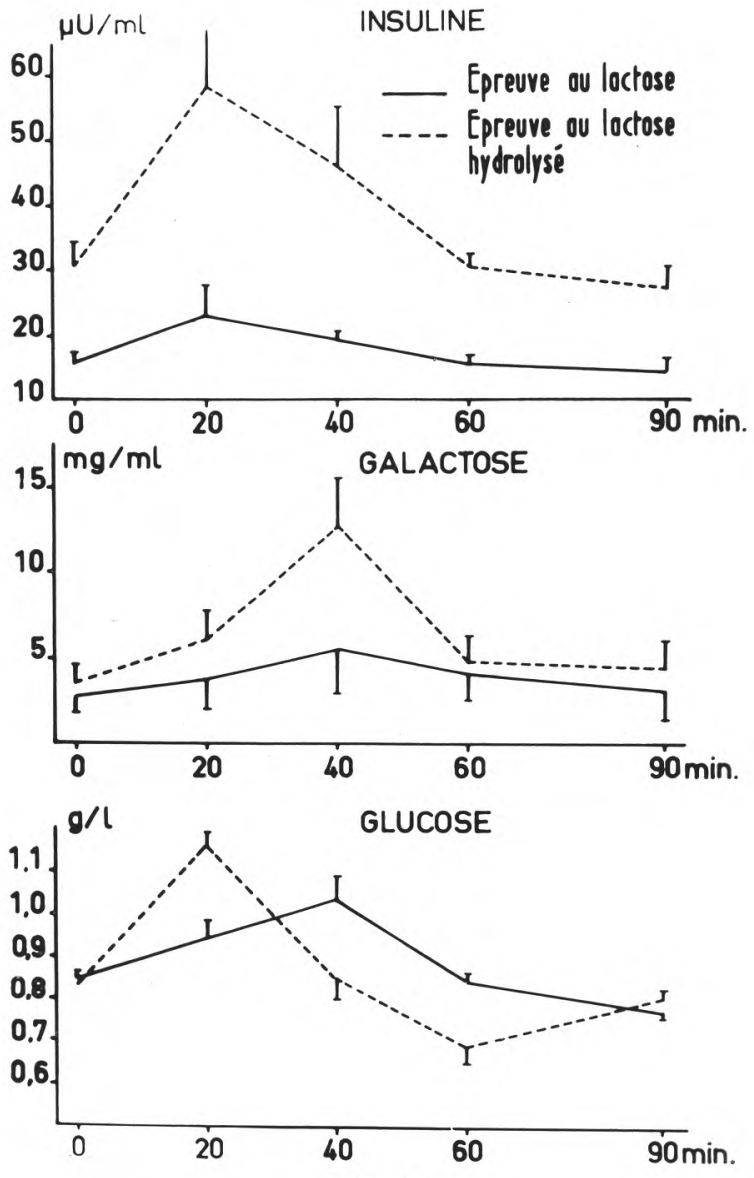

fig. 1

Variations de la glycémie, de la galactosémie et de l'insulinémie au cours des deux épreuves.

\subsection{Glucose sanguin}

Le pic d'hyperglycémie se situe pour les trois sujets $40 \mathrm{mn}$ après l'ingestion lors de la première épreuve (lactose pur) et $20 \mathrm{mn}$ seulement après ingestion de lactose hydrolysé. 
TABLEAU 2. - Test au lactose solution pure

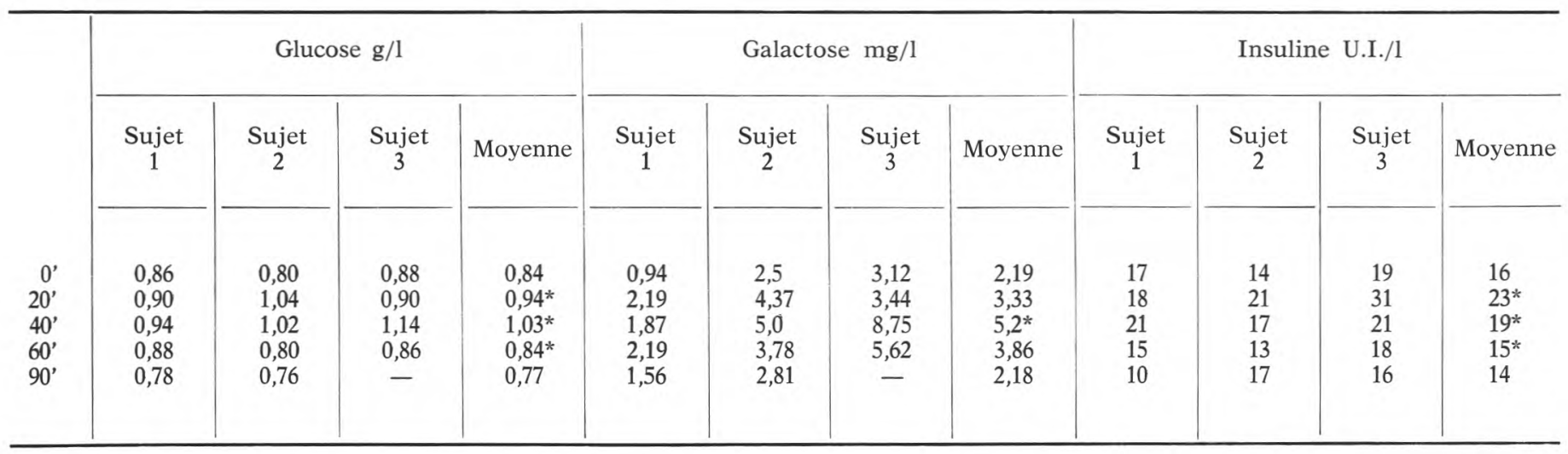

Test au lactose hydrolysé

\begin{tabular}{|c|c|c|c|c|c|c|c|c|c|c|c|c|}
\hline & \multicolumn{4}{|c|}{ Glucose $\mathrm{g} / \mathrm{l}$} & \multicolumn{4}{|c|}{ Galactose $\mathrm{mg} / \mathrm{l}$} & \multicolumn{4}{|c|}{ Insuline U.I./1 } \\
\hline & $\underset{1}{\text { Sujet }}$ & $\underset{2}{\text { Sujet }}$ & $\underset{3}{\text { Sujet }}$ & Moyenne & $\underset{1}{\text { Sujet }}$ & $\underset{2}{\text { Sujet }}$ & $\underset{3}{\text { Sujet }}$ & Moyenne & $\underset{1}{\text { Sujet }}$ & $\underset{2}{\text { Sujet }}$ & $\underset{3}{\text { Sujet }}$ & Moyenne \\
\hline $\begin{array}{l}0^{\prime} \\
20^{\prime} \\
40^{\prime} \\
60^{\prime} \\
90^{\prime}\end{array}$ & $\begin{array}{l}0,82 \\
1,14 \\
0,94 \\
0,70 \\
0,78\end{array}$ & $\begin{array}{l}0,84 \\
1,12 \\
0,88 \\
0,74 \\
0,84\end{array}$ & $\begin{array}{l}0,84 \\
1,24 \\
0,74 \\
0,74 \\
0,78\end{array}$ & $\begin{array}{l}0,83 \\
1,16^{*} \\
0,85^{*} \\
0,69^{*} \\
0,80\end{array}$ & $\begin{array}{c}1,56 \\
2,81 \\
15,31 \\
7,5 \\
7,81\end{array}$ & $\begin{array}{r}3,78 \\
6,25 \\
15,62 \\
3,78 \\
2,81\end{array}$ & $\begin{array}{l}5,0 \\
9,37 \\
7,81 \\
2,19 \\
1,87\end{array}$ & $\begin{array}{r}3,44 \\
6,14 \\
12,9^{*} \\
4,49 \\
4,16\end{array}$ & $\begin{array}{l}37 \\
65 \\
50 \\
30 \\
33\end{array}$ & $\begin{array}{l}30 \\
72 \\
29 \\
28 \\
27\end{array}$ & $\begin{array}{l}28 \\
37 \\
60 \\
35 \\
21\end{array}$ & $\begin{array}{l}31 \\
58^{*} \\
46^{*} \\
31^{*} \\
27\end{array}$ \\
\hline
\end{tabular}

* Différence significative (test de Student $\mathrm{P}<0,05$ ). 


\subsection{Galactose sanguin}

Lors de l'épreuve au lactose le pic de galactosémie se situe $40 \mathrm{mn}$ après l'ingestion du produit.

Après l'absorption du lactose hydrolysé on observe une augmentation de la galactosémie sanguine avec un taux maximum après $40 \mathrm{mn}$. Les valeurs de galactosémie sont plus élevées lors de l'épreuve au lactose hydrolysé.

\subsection{Insuline}

L'évolution de l'insuline lors des deux épreuves est corrélée à celle de la glycémie. L'augmentation relative maximum de l'insulinémie après $20 \mathrm{mn}$ est plus faible lors de la première épreuve ( $43 \mathrm{p}, 100$ ) que lors de la seconde épreuve où l'augmentation avoisine 90 p. 100.

\section{DISCUSSION}

Au cours de la première épreuve le niveau basal d'insuline varie peu après l'ingestion du lactose pur, l'absorption de celui-ci se faisant lentement.

Au cours de la seconde épreuve le niveau basal d'insuline est doublé $1 \mathrm{~h}$ après l'ingestion du lactose hydrolysé. Lefèvre (1976) constate après un petit déjeuner complet un niveau d'insuline comparable.

Cette réponse physiologique à l'absorption du lactose hydrolysé peut donc être considérée comme normale compte tenu des quantités ingérées semblables à celles de lactose absorbées dans un petit déjeuner traditionnel.

En outre lors de l'ingestion du lactose hydrolysé au cours d'un petit déjeuner complet (en association avec des lipides et des protéines), l'absorption des sucres simples sera retardée par la compétition avec les acides aminés au niveau de la barrière intestinale. (Rossiter, 1974).

En tout état de cause, les conséquences de l'introduction de l'hydrolysat de lactose dans l'alimentation humaine doivent être envisagées en fonction de son contexte d'utilisation, et du produit qu'il remplace (type de sucre substitué, quantité introduite, nutriments associés dans le produit).

En conclusion, outre des applications industrielles comme édulcorant, cette matière première peut servir de base à des produits diététiques destinés aux malades présentant une intolérance congénitale ou passagère au lactose, ainsi qu'aux sujets subissant une réalimentation progressive après une médication ou une intervention chirurgicale ayant entraîné une dégradation des fonctions digestives de la muqueuse intestinale. 


\section{S u $\mathrm{m} \mathrm{m}$ a r y}

ABSORPTION OF LACTOSE AND HYDROLYSED LACTOSE IN MAN

In prospect to introduce the hydrolysed lactose in human nutrition we study the effect of lactose substitution by its constitutive sugars (glucose and galactose) on the absorption of these sugars and the stimulation of insuline secretion.

\section{Bibliographie}

Bondy (P. K.), Rosenberg (L. E.) (1974), - In Duncan « Diseases of Metabolism ». Ed. W. B. Saunders Company, Philadelphia (U.S.A.), 318-320.

Bunce (G. E.), Hess (J.L.) (1976). - Lenticular opacities in young rats as a consequence of maternal diets low in tryptophan and/or vitamin E. J. Nutr., $106,222-229$.

GaRga (C.), Scrimshaw (N.S.) (1976). - Relationship of lactose intolerance to milk intolerance in young children. Amer. J. Clin. Nutr., 29, 192-196.

GuY (E.), TAMSMa (A.), Konston (A.), Holsinger (V.) (1974). - Lactose-treated milk provides base to develop products for lactose- intolerant populations. Food Prod. Develop., 8, 50-74.

Hales (C. N.), Randle (P. J.) (1963). - Immunoassay of insulin with insulin antibody precipitate. Biochem J., 88, 137-146.

HefFley (J. D.), Williams (R. J.) (1974). - The nutritional teamwork approach: prevention and repression of cataracts in rats. Proc. Nat. Acad. Sci., 10, 4164-4168.

Jones (D. V.), Lathan (M. C.) (1976). - Symptom response to lactose reduced milk in lactose intolerant adults. Amer. J. Clin. Nutr., 29, 633-638.

Jones (D. V.), Lathan (M. C.) (1974). - Lactose intolerance in young children and their parents. Amer. J. Clin. Nutr., 27, 547-549.

LeFEBVRE (P. J.), LUY'KX (A.S.) (1976). - The breakfast tolerance test a return to physiology. Diabète et métabolisme, 2, 15-19.

Marschner (I.), Bottermann (P.), ERhardt (F.), Linke (R.), LofFler (G.), Maier (V.), Schwandt (P.), Vogt (W.), Scriba (P.C.) (1974). - Group experiments on the radioimmunological insulin determination. Horm. Metab. Res., 6, 293-296.

Morgan (C. R.), Lazarow (A.) (1963). - Immunoassay of insulin: two antibody system. Diabetes, 12, 115-126.

Paige (D. M.), Bayless (T. M.), Huang (S. S.), Wexler (R.) (1975). - Lactose hydrolysed milk. Am. J. Clin. Nutr., 28, 818-922.

Paige (D. M.), Bayless (T. M.), Dellinger (W. S.) (1975). - Relationship of milk consumption to blood glucose rise in lactose intolerant individuals. Am. J. Clin. Nutr., 28, 677-680.

Rodger (F. C.), Sinclair (H. M.) (1969). - Metabolic and Nutritional eye diseases. Ed. Charles C. Thomas, Springfield (Illinois - U.S.A.), 100-102.

Roger (L.), Thapon (J. L.), Maubors (J. L.), Brule (G.) (1976). - Hydrolyse du lactose contenu dans l'ultrafiltrat de lait ou de lactosérum en réacteur enzymatique à membrane. Le Lait, 56, 56-75. 
Rossiter (M. A.), Palmer (T.), Evans (K.), Whartow (B. A.) (1974). - The short term response to a drink of milk, lactose or casein in children with apparently normal gastrointestinal tracts. Br.J. Nutr., 32, 6C5-613.

SAHI (T.) (1974). - The inheritance of selective adult-type lactose malabsorption. Scand. J. Gastroent., 9, sup. 30, 29-30.

Seetharam Bhat (K.), Gopalan (G.) (1974). - Human cataract and galactose metabolism. Nutr. Metab., 17, 1-8.

Turner (S. J.), Daly (T. H.), Hourrigan (J. A.), Rand (A.), Thayer (W. R.) (1976). Utilisation of a low-lactose milk. Amer. J. Clin. Nutr., 29, 739-745.

VARMa (S. N.), Schwarz (V.) (1974). - Does dietary galactose play a part in the synthesis of brain lipids of the newborn? Biochem. J., 43, 1960.

WAAR (D. H.) (1975). - Zu wirkung von lactose in der Nahrung auf das wachstum und die gehirnent-wicklungsprotenz bei Ratten. Z - ernährungsw., 4, 189-199. 